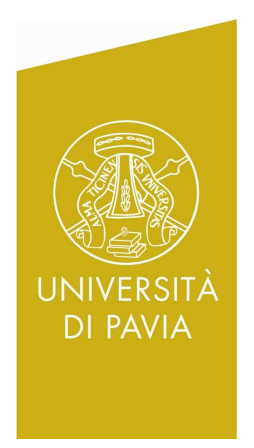

Department of Economics and Management DEM Working Paper Series

\title{
Monopolistic Competition: A Dual Approach
}

\author{
Paolo Bertoletti \\ (Università di Pavia) \\ Federico Etro \\ (Università Ca' Foscari di Venezia) \\ \# 43 (05-13) \\ Via San Felice, 5 \\ I-27100 Pavia \\ http://epmq.unipv.eu/site/home.html
}




\title{
Monopolistic Competition: A Dual Approach
}

\author{
Paolo Bertoletti and Federico Etro ${ }^{1}$ \\ University of Pavia and Ca' Foscari University of Venice
}

First version: December 2012

Current version: April 2013

Key words: Monopolistic competition, Indirect additivity, Dixit-Stiglitz model, Endogenous entry

JEL Codes: D11, D43, L11, F12

\begin{abstract}
We study monopolistic competition under indirect additivity of preferences. This is dual to the Dixit-Stiglitz model, where direct additivity is assumed, with the CES case as the only common ground. Other examples include (perceived) demand functions that are exponential or linear. Our equilibrium results are generally in contrast with those received by the literature. An increase of the number of consumers never affects prices and firms' size, but increases proportionally the number of firms, creating pure gains from variety. An increase in individual income increases prices (and more than proportionally the number of varieties) and reduces firms' size if and only if the price elasticity of demand is increasing. We also study the endogenous market structure with Bertrand competition (in which a pro-competitive effect of market size arises) and the case for inefficient entry.

\footnotetext{
${ }^{1}$ We are grateful to Paolo Epifani and Kresimir Zigic for insightful comments on an early version of the paper. Correspondence. Paolo Bertoletti: Dept. of Economics and Management, University of Pavia, Via San Felice, 5, I-27100 Pavia, Italy. Tel: +390382 986202, email: paolo.bertoletti@unipv.it. Federico Etro: Dept. of Economics, University of Venice Ca' Foscari, Sestiere Cannaregio, 30121, Fond.ta S.Giobbe 873, Venice, Italy. Tel: +39-0412349172, email: federico.etro@unive.it.
} 
The Dixit and Stiglitz (D-S, 1977) model of monopolistic competition and endogenous entry à la Chamberlin (1933) has been widely applied in the modern theories of trade and in macroeconomics. Due to its analytical tractability, most applications of the D-S setting rely on the particular case of CES preferences, which generates equilibrium prices and output levels that are independent both from the number of consumers (the market "size") and from the level of individual income. However, since Krugman (1979) the general model has been used to study the "competitive effects" on firm behaviour created by changes in the number of consumers: for a recent discussion see Zhelobodko et al. (2012) and Bertoletti and Epifani (2012).

The D-S setting assumes "direct additivity", i.e., that consumers' preferences can be represented by an additively separable utility function. In this note, by assuming "indirect additivity", i.e., that consumers' preferences can be represented by an additively separable indirect utilitity function, we develop an alternative model of monopolistic competition, and characterize its equilibrium and welfare properties. Notice that indirect additivity amounts to assume that the relative demand of two goods does not depend on the price of other goods. This is "dual" to, and at least as reasonable as, the assumption of direct additivity made in the "primal" D-S approach, for which the marginal rate of substitution between any two goods does not depend on the consumption of other goods. Most important, the case of CES preferences is the only common ground of these two forms of separability. Therefore our analysis applies to an (almost) entirely different class of preferences than D-S. It is also worth noticing that preferences which satisfy indirect additivity generate analytically tractable direct demand functions: examples include isoelastic, exponential and linear "perceived" demands.

Perhaps surprisingly, our results are rather different from those received by the 
literature. In particular, indirect additivity implies that an increase in the number of consumers never affects equilibrium prices and firm size, and just expands linearly the number of goods produced, so as to generate pure welfare gains from variety. Accordingly, these crucial properties of the CES case extend to a wide class of (nonhomothetic) preferences. This result is reassuring for the many trade models based on CES preferences (e.g., Krugman, 1980 and Melitz, 2003), and in striking contrast with the general D-S setting, whereby the competitive effects of market size, and even welfare gains, depend ambiguously on the properties of what Zhelobodko et al. (2012) call the "relative love for variety" embedded into preferences.

Moreover, in our setting preferences directly determine demand elasticity and naturally allow income changes to affect it. If, for instance, demand elasticity is increasing in the price (arguably the normal case), a rise of individual income makes demand more rigid, inducing higher prices, a reduction of firm size and a more than proportional increase in the free-entry equilibrium number of firms. This is again in contrast with the result of the D-S setting, whereby equilibrium prices are independent from income, whose growth only expands proportionally the number of varieties. Such a "neutrality" simplifies a lot the macroeconomic analysis of technological progress/shocks in applied work (for a recent application see Bilbiie et al., 2012), but it should not be expected to hold unless preferences are homothetic. In addition, in our setting a productivity shock affecting marginal costs can be translated to prices more or less than proportionally, and so it affects entry. That is, contrary to what happens with CES preferences, supply shocks change the market structure generating additional processes of business creation/destruction, which could alter macroeconomic reactions.

We also briefly consider the welfare properties of free-entry equilibria in our set- 
ting, emphasizing the conditions for inefficient entry and analyzing the impact of growth on welfare. Finally, we generalize our analysis to endogenous market structures in which firms compete $\grave{a}$ la Bertrand, and thus a competitive effect of market size is restored. We also discuss a number of implications due to the simple aggregative nature of this class of games.

The work is organized as follows. Section 1 presents our model of monopolistic competition and characterizes its equilibrium. In Section 2 we consider the case for inefficient entry in our setting. Finally, we study Bertrand competition in Section 3 and conclude in Section 4.

\section{The Model}

Consider $L$ identical agents consuming $n$ goods under the following, symmetric, indirect utility function:

$$
V(\mathbf{p}, E)=\Psi\left(\sum_{j=1}^{n} v\left(\frac{p_{j}}{E}\right)\right),
$$

where $E>0$ is the income of each agent to be spent in the differentiated goods, ${ }^{2}$ $\mathbf{p}>0$ is the price vector and $\Psi$ is a monotonic increasing and differentiable transformation. The expression on the RHS of (1) exploits the homogeneity of degree zero of the indirect utility, and crucially assumes additive separability (i.e., "indirect additivity"). To satisfy sufficient conditions for (1) being an indirect utility function while allowing for a possibly finite choke-off price $\bar{s}$, we assume that $v(s)$ is at least thrice differentiable, with $v^{\prime \prime}(s)>0>v^{\prime}(s)$ for $s<\bar{s}$, and that $\lim _{s \rightarrow \bar{s}} v(s), v^{\prime}(s)=0$, with

\footnotetext{
${ }^{2}$ Using the wage as the numeraire, $E$ can be interpreted as the labor endowment of each agent (in efficiency units).
} 
$v(s)=0$ for $s \geq \bar{s}$ (these assumptions imply that demand and extra utility are zero for a good that is not produced, i.e., that has a high enough price).

The Roy identity generates the following (Marshallian) direct demand function of each consumer for good/variety $i=1, \ldots, n$ :

$$
x_{i}(\mathbf{p}, E)=\frac{v^{\prime}\left(\frac{p_{i}}{E}\right)}{\sum_{j=1}^{n} v^{\prime}\left(\frac{p_{j}}{E}\right) \frac{p_{j}}{E}},
$$

which delivers the total market demand $q_{i}=x_{i}(\mathbf{p}, E) L$. Notice that in the RHS of $(2)$ the expression at the denominator, say $\mu(\mathbf{p}, E)<0$, is (up to a monotonic tranformation) the negative of the marginal utility of income, times the income/expenditure level $E$.

Examples of (1) include simple cases such as the isoelastic function $v(s)=s^{1-\theta}$ with $\theta>1$, the case of "mixtures" as $v(s)=s^{1-\alpha}+s^{1-\theta}$ with $\alpha>1, \alpha \neq \theta$, the negative exponential function case with $v(s)=e^{-\tau s}$ and $\tau>0$, and the "addilog" function $v(s)=(a-s)^{1+\gamma}$ with $a, \gamma>0 .{ }^{3}$ Note that only if $v(\cdot)$ is isoelastic preferences are homothetic. Indeed, in such a case they are of the CES type, with indirect utility $V(\mathbf{p}, E)=E\left(\sum_{j=1}^{n} p_{j}^{1-\theta}\right)^{1 /(1-\theta)}$ and $\theta$ equal to the elasticity of substitution. By an important duality result (see Hicks, 1969 and Samuelson, 1969), the case of CES preferences is the only one in which the class of preferences (1) satisfies direct addivitity. That is, it is the only case in which preferences can also be represented by an additively separable direct utility function, $U(\mathbf{x})=F\left(\sum_{j=1}^{n} u\left(x_{j}\right)\right)$, as assumed in the D-S model. Therefore, the indirect utility (1) encompasses a class of (nonhomothetic) preferences whose corresponding direct utility functions are non-additive.

To compare the assumptions of direct (primal) and indirect (dual) additivity, notice that indirect additivity amounts to assume that the consumption ratio of any two

\footnotetext{
${ }^{3}$ Here the choke-off price $\bar{s}=a$ can be made arbitrary large.
} 
goods $i$ and $j, x_{i}(\mathbf{p}, E) / x_{j}(\mathbf{p}, E)$, does not depend on the price of any other good, a rather intuitive concept (and also a reasonable approximation for classes of goods that are well differentiated). Under the primal approach, on the contrary, it is the marginal rate of substitution between any two goods, $M R S_{i, j}(\mathbf{x})=\left(\partial U(\mathbf{x}) / \partial x_{i}\right) /\left(\partial U(\mathbf{x}) / \partial x_{j}\right)$, which is independent of the consumption of other goods, leading to the dual property that their "inverse" price ratio, $p_{i}(\mathbf{x}) / p_{j}(\mathbf{x})$, is independent from the quantities of the other goods. If both these properties are assumed to hold, then symmetric preferences must be of the CES type (see e.g. Blackorby et al., 1978, Section 4.5.3).

Suppose now that each variety is sold by a (symmetric) firm producing with constant marginal cost $c>0$ and fixed cost $F>0 .{ }^{4}$ Following D-S, we model monopolistic competition by assuming that there are so many varieties that the impact of each individual price on the marginal utility of income is negligible. ${ }^{5}$ Accordingly, the direct demand function "perceived" by firm $i$ in monopolistic competition is given by $q_{i}=v^{\prime}\left(p_{i} / E\right) L / \mu$, where $\mu$ is taken as given, and its profit can be written as:

$$
\pi\left(p_{i}, E\right)=\frac{\left(p_{i}-c\right) v^{\prime}\left(\frac{p_{i}}{E}\right) L}{\mu}-F .
$$

The most relevant implication of this functional form is that the perceived demand elasticity corresponds to the (absolute value of the) elasticity of $v^{\prime}(\cdot)$, which we define as $\theta(s) \equiv-v^{\prime \prime}(s) s / v^{\prime}(s)>0$. Notice that $\theta$ can be interpreted as the elasticity of substitution between any two varieties when their prices are both equal to $s$ (see the Appendix). Moreover, it depends on the price as a fraction of income, $p_{i} / E$, but is independent of $\mu$ and $L$. Instead, in the primal case analyzed by D-S, the

\footnotetext{
${ }^{4}$ As in Krugman (1980), where labor is used to produce goods and firms, by normalizing the wage to unity, $c$ and $F$ can be thought as expressed in term of labor units.

${ }^{5}$ Formally, the elasticity of $\mu$ with respect to $p$ is of order $1 / n$ if prices are not disproportionate: see the Appendix.
} 
perceived elasticity of inverse demand is uniquely determined by the consumption level. ${ }^{6}$ This difference will be crucial for the analysis of the monopolistic competition equilibrium because market adjustments (needed to restore the zero-profit condition of endogenous entry) take place through shifts of perceived demands due to changes in the number of firms which affect the marginal utility of income.

Firm $i$ maximizes (3) with respect to $p_{i}$. Its optimal price must then satisfy the FOC:

$$
v^{\prime}\left(\frac{p_{i}}{E}\right)+\frac{\left(p_{i}-c\right) v^{\prime \prime}\left(\frac{p_{i}}{E}\right)}{E}=0
$$

with SOC $2 v^{\prime \prime}+\left(p_{i}-c\right) v^{\prime \prime \prime} / E>0$. To satisfy (4) we assume that (locally) $v^{\prime \prime} s+v^{\prime}>0$, which implies $\theta>1$ (this is equivalent to assume that goods are gross substitutes). If demand is (locally) concave $\left(v^{\prime \prime \prime}>0\right)$ the SOC is satisfied and $\theta^{\prime}>0$, that is, demand is perceived as more elastic when the price goes up. On the contrary, if demand is convex ( $v^{\prime \prime \prime}<0$, as in the CES case) we may have $\theta^{\prime}<0$, that is, demand is perceived as less elastic when the price increases. Notice that an equivalent (local) condition for the SOC to be satisfied is $2 \theta>\zeta \equiv-v^{\prime \prime \prime} s / v^{\prime \prime}$, where $\zeta$ is a measure of curvature of perceived demand.

The FOC (4) can be rewritten as follows for the optimal price $p^{e}$ (we omit in what follows the suffix $i$ to refer to a firm/variety):

$$
\frac{p^{e}-c}{p^{e}}=\frac{1}{\theta\left(\frac{p^{e}}{E}\right)}
$$

where the profit maximizing value of the Lerner index, the RHS of (5), is given by the reciprocal of the perceived demand elasticity. To guarantee the existence of a

\footnotetext{
${ }^{6}$ In the D-S model the perceived (individual) inverse demand of variety $i$ is given by $p_{i}\left(x_{i}\right)=$ $u\left(x_{i}\right) / \lambda$, where $\lambda$ is the marginal utility of income (up to a monotonic transformation). Its elasticity is provided by $\sigma\left(x_{i}\right)=-u^{\prime}\left(x_{i}\right) /\left(u^{\prime \prime}\left(x_{i}\right) x_{i}\right)$, which can be interpreted as the elasticity of substitution between varieties $i$ and $j$ if $x_{i}=x_{j}$ : see Bertoletti and Epifani (2012).
} 
solution to (5) we assume that $\bar{s} E>c$ (so that consumer willingness to pay is large enough) and that $\lim _{s \rightarrow \bar{s}} \theta(s)>\bar{s} E /(\bar{s} E-c)$.

The price rule (5) says that under indirect additivity the optimal price is always independent from the number of varieties supplied, because this does not affect the perceived demand elasticity. The comparative statics results with respect to $E$ and $c$, however, fully depend on the sign of $\theta^{\prime}$. For instance, if $\theta^{\prime}>0$, the optimal price grows with income because firms face a more rigid demand (perhaps an entirely natural result). Similarly, it is easy to verify that a change in the marginal cost $c$ is transmitted (pass-through) to prices in a less than proportional way (undershifting) if $\theta^{\prime}>0$, and in a more than proportional way (overshifting) if $\theta^{\prime}<0$. Notice that in the D-S model the optimal price depends, on the contrary, on the consumption level through an index of "relative love for variety" (Zhelobodko et al., 2012), and therefore on the number of firms. ${ }^{7}$

Since by symmetry the equilibrium profit is the same for all firms, and it is decreasing in their number, we can characterize the endogenous market structure thorugh the following zero profit condition: ${ }^{8}$

$$
\frac{(p-c) E L}{n p}=F
$$

This and the pricing rule (5) deliver the free-entry number of firms and firm size:

$$
n^{e}=\frac{E L}{F \theta\left(\frac{p^{e}}{E}\right)}, \quad q^{e}=F \frac{\theta\left(\frac{p^{e}}{E}\right)-1}{c} .
$$

Notice that the equlibrium number of firms $n^{e}$ is proportional to $L / F$, while $q^{e}$ is proportional to $F$. These are well-known results of monopolistic competition with

\footnotetext{
${ }^{7}$ The price rule of the D-S model is $(p-c) / p=1 / \sigma(x)$, where $1 / \sigma(x)$ is the "relative love for variety" and $x=E / p n$ by the budget constraint (therefore $n$ affects the price).

${ }^{8} \mathrm{As}$ it is standard in the literature, in what follows we treat $n$ as a real number.
} 
CES preferences that generalize to the whole class of preferences described by (1). In terms of elasticities, indirect additivity implies (with obvious notation):

$$
\epsilon_{p L}=\epsilon_{q L}=0 \quad \text { and } \quad \epsilon_{n L}=1
$$

which is in striking contrast to what emerges in the D-S model, where the comparative statics results of changes in $L$ depend on the type of preferences. ${ }^{9}$ Therefore, the classic implications of trade models à la Krugman (1980) with CES preferences are valid for all preferences satisfying indirect additivity: opening up to trade creates pure gains from variety without any competitive effect on prices and firm size.

We conclude the comparative statics analysis of our market structure deriving the impact of changes in individual income and marginal cost:

$$
\begin{gathered}
\epsilon_{p E} \gtrless 0, \epsilon_{q E} \lessgtr 0, \text { and } \epsilon_{n E} \gtrless 1 \text { iff } \theta^{\prime}\left(p^{e} / E\right) \gtrless 0, \\
\epsilon_{p c} \lessgtr 1, \epsilon_{q c} \gtrless-1 \text { and } \epsilon_{n c} \lessgtr 0 \text { iff } \theta^{\prime}\left(p^{e} / E\right) \gtrless 0 .
\end{gathered}
$$

Accordingly, increasing individual income has an ambiguous effect on prices and firm size, depending on its impact on demand elasticity. The intuition is that when higher income makes demand more rigid $\left(\theta^{\prime}>0\right)$, firms increase their prices and restrict production, which promotes business creation and increases the number of firms more than proportionally. Incidentally, our model provides a simple rationale for pricingto-market: prices should be higher for richer markets. Once again these result are

\footnotetext{
${ }^{9}$ The free-entry equilibrium conditions of the D-S model can be summarized as follows:

$$
\frac{p^{e}-c}{p^{e}}=\frac{1}{\sigma\left(x^{e}\right)}, \quad n^{e}=\frac{E L}{F \sigma\left(x^{e}\right)} \quad \text { and } q^{e}=F \frac{\sigma\left(x^{e}\right)-1}{c} .
$$

Notice that $\sigma$ replaces $\theta$ in the formulas, with dual consequences. One can verify that the impact of $L$ depends on the sign of $\sigma^{\prime}$ (see Zhelobodko et al., 2012). On the contrary, $E$ is neutral on price and firm size, and increases the number of varieties proportionally.
} 
in contrasts with the D-S setting, where income growth is inconsequential on firm behavior, and only increases proportionally the equilibrium number of firms. ${ }^{10}$

Last, notice that since a change in $c$ may affect prices more or less than proportionally, the marginal cost has an ambiguous impact on the number of firms which depends on the pattern of demand elasticity. For example, when demand elasticity is increasing $\left(\theta^{\prime}>0\right)$, lower marginal costs are translated less than proportionally to prices, which increases the markups and attracts entry of new firms. Accordingly, and contrary to what happens with CES preferences, our general model suggests that demand shocks (i.e., affecting expenditure) and supply shocks (i.e., affecting marginal cost) generate additional processes of business creation/destruction. This should alter the dynamics of macroeconomic models with endogenous entry and monopolistic competition (Bilbiie et al., 2012) or oligopolistic competition (Etro and Colciago, 2010).

Our results can be illustrated in the following examples. The first is based on the (negative) exponential function $v(s)=e^{-\tau s}$, which generate the perceived demand $q_{i}=\tau e^{-\tau p_{i} / E} L /(-\mu)$. The free-entry equilibrium implies:

$$
p^{e}=c+\frac{E}{\tau}, \quad n^{e}=\frac{E^{2} L}{F(c \tau+E)}, \quad q^{e}=\frac{F \tau}{E} .
$$

The second example refers to the addilog case $v(s)=(a-s)^{1+\gamma}$, which delivers a linear perceived demand $q_{i}=2\left(a-p_{i} / E\right) L /(-\mu)$ when $\gamma=1$. For the general case we obtain the following equilibrium values:

$$
p^{e}=\frac{\gamma c}{1+\gamma}+\frac{a E}{1+\gamma}, \quad n^{e}=\frac{(a E-c) E L}{F(a E+\gamma c)}, \quad q^{e}=\frac{F(1+\gamma)}{a E-c} .
$$

\footnotetext{
${ }^{10}$ The reason of these different results is rooted in the market adjustment process. Since the profit expression in the primal approach is $\pi=\left(u^{\prime}(x) / \lambda-c\right) L x-F$, where $\lambda=\sum_{j} u^{\prime}\left(x_{j}\right) x_{j} / E$, there is a unique (symmetric) equilibrium (zero-profit) value of $\lambda=\left(n u^{\prime}(x) x\right) / E$. On the contrary, under indirect additivity, there is a unique equilibrium value of $L / \mu=L E /\left[n v^{\prime}(p / E) p\right]$.
} 
Notice that in both examples $\theta^{\prime}>0$ : therefore, growth in income makes demand more rigid, which leads firms to increase their prices and reduce their production, with a more than proportional increase in the number of firms. In addition, a marginal cost reduction is not fully translated to the prices, which attracts more business creation and has a limited impact on firm size: notice that $q^{e}$ is unchanged in the exponential case and decreases in the addilog case.

In conclusion, it may be useful to have an idea of the kind of direct utility functions which satisfy indirect additivity. This can be done by using the Roy identity to obtain the inverse demand $p_{i}(\mathbf{x})=E v^{\prime-1}\left(\mu x_{i}\right)$ for each variety $i$. Employing the budget constraint $\sum_{j=1}^{n} p_{i}(\mathbf{x}) x_{i}=E$, we obtain that $\mu$ is implicitly defined from $1=\sum_{j=1}^{n} v^{\prime-1}\left(\mu x_{j}\right) x_{j}$. A close-form solution is available for our examples. With the exponential demand we obtain:

$$
p_{i}(\mathbf{x})=\frac{E}{\tau}\left[\ln (-\mu)^{-1}-\ln x_{i}\right], \quad \text { where } \mu=-e^{-\frac{\tau+\sum_{j=1}^{n} x_{j} \ln x_{j}}{\sum_{j=1}^{n} x_{j}}}
$$

and in the addilog case we have:

$$
p_{i}(\mathbf{x})=E\left[a-x_{i}^{1 / \gamma}\left(\frac{-\mu}{1+\gamma}\right)^{1 / \gamma}\right], \text { where } \mu=-(1+\gamma)\left[\frac{a \sum_{j=1}^{n} x_{j}-1}{\sum_{j=1}^{n} x_{j}^{\frac{1+\gamma}{\gamma}}}\right]^{\gamma}
$$

Of course, our previous results could be re-derived by assuming that each firm $i$ takes $\mu$ as given and chooses its production level $x_{i}$ to maximize $\pi_{i}=\left(p_{i}(\mathbf{x})-c\right) L x_{i}-F$. Finally, we can solve for the direct utility functions by plugging the inverse demand in the indirect utility (1). In the two examples we obtain respectively:

$$
U(\mathbf{x})=F\left[\sum_{j=1}^{n} x_{i} \exp \left(-\frac{\tau+\sum_{j=1}^{n} x_{j} \ln x_{j}}{\sum_{j=1}^{n} x_{j}}\right)\right]
$$


and

$$
U(\mathbf{x})=F\left[\frac{\left(a \sum_{j=1}^{n} x_{j}-1\right)^{1+\gamma}}{\left(\sum_{j=1}^{n} x_{j}^{\frac{1+\gamma}{\gamma}}\right)^{\gamma}}\right] .
$$

Notice in these expressions the role of two simple aggregators of the consumption levels: total consumption and an index of consumption dispersion.

\section{Optimality and inefficient entry}

As well known, endogenous entry and product differentiation tend to bias the allocation of resources compared to what a social planner would choose. Firms do not fully internalize the impact of their entry decisions on the profits of competitors (a business stealing effect) and on the gains from variety (a non-appropriability effect), which may lead to too many or too few firms. Let us consider the optimal allocation of resources in our setting. This solves the following problem:

$$
\max _{n, p} n v\left(\frac{p}{E}\right)
$$

under the resource constraint $E L \geq n(c q+F)=n(c E L / n p+F)$, which is equivalent to (6). The FOCs ${ }^{11}$ can be rearranged as follows:

$$
\frac{p^{*}-c}{p^{*}}=\frac{1}{1+\eta\left(\frac{p^{*}}{E}\right)}, \quad n^{*}=\frac{E L}{F\left[1+\eta\left(\frac{p^{*}}{E}\right)\right]},
$$

where $\eta(s) \equiv-v^{\prime}(s) s / v(s)>0$ is the absolute value of the elasticity of $v(\cdot)$. To guarantee the existence of a solution to the equations in (10) we assume that $\lim _{s \rightarrow \bar{s}} \eta(s)>$ $c /(\bar{s} E-c)$. From the comparison of $(5),(7)$ and (10) we see that an excess of entry arises (i.e., $\left.n^{e}>n^{*}\right)$ if and only if $p^{*}<p^{e}$ and $\theta\left(p^{e} / E\right)<\eta\left(p^{*} / E\right)+1$. It is also easily computed that:

\footnotetext{
${ }^{11}$ We assume that they characterize the optimal allocation.
} 


$$
\eta^{\prime}(s)=\frac{\eta(s)[1-\theta(s)+\eta(s)]}{s}
$$

whose sign is ambiguous in general. However, in the CES case $\eta=\theta-1$ and thus the free entry equilibrium is socially optimal, as known from D-S: the business stealing and non-appropriability effects balance each other.

Consider now the general case: since it follows from (11) that $\eta^{\prime} \lessgtr 0$ is equivalent to $1 /(1+\eta(p / E)) \gtrless 1 / \theta(p / E),(5)$ and (10) imply that (globally) $\eta^{\prime} \lessgtr 0$ is equivalent to $n^{e} \lessgtr n^{*}$, a result which extends to our setting the possibility of inefficient entry under monopolistic competition. ${ }^{12}$ Paralleling D-S (p. 303), an intuition for this result can be obtained by noticing that $\eta$ is approximately the ratio between the revenue of each firm and the additional utility generated by its variety. If $\eta^{\prime}>(<) 0$ they diverge and at the margin each firm finds it more profitable to price higher (lower), i.e, to produce less (more), than what would be socially desirable. This, in turn, attracts too many (too few) firms.

It is also worth noticing that in our setting an increase of $E$ always decreases $p^{e} / E$ (as it can be seen by differentiating (4)). If it also increases $n^{e}$, as it is necessarily the case if $\theta^{\prime} \geqslant 0$, then welfare $\Psi\left(n^{e} v\left(p^{e} / E\right)\right)$ improves. However, we cannot exclude the case of immiserizing growth, for which it can be proved that a necessary condition is $\eta^{\prime}<0 .{ }^{13}$ That is, an income rise can be welfare deproving only if it causes a further reduction in the number of varieties when there is already insufficient entry.

\footnotetext{
${ }^{12}$ Notice that $\eta^{\prime}>0$ for the negative exponential and addilog cases: accordingly, they both imply an excess of free entry.

${ }^{13}$ Computation shows that a rise of income decreases consumers welfare if and only if $2 \theta-\zeta+1+$ $\frac{\eta^{\prime} s}{\eta}<0$ (notice that the SOC for profit maximization implies that $2 \theta>\zeta$ ).
} 


\section{Endogenous market structures with Bertrand competition}

When the number of firms is small, strategic interactions play a relevant role: here we analyze this possibility focusing on Bertrand competition and endogenous entry. ${ }^{14}$ Considering the actual Marshallian demand, each firm $i$ chooses its price $p_{i}$ to maximize profit:

$$
\pi\left(p_{i}, E\right)=\left(p_{i}-c\right) x_{i}(\mathbf{p}, E)-F
$$

where $x_{i}(\mathbf{p}, E)$ is given by (2). In a symmetric Bertrand equilibrium demand elasticity is easily computed (see the Appendix) as:

$$
-\frac{\partial x(\mathbf{p}, E)}{\partial p} \frac{p}{x(\mathbf{p}, E)}=\frac{\theta\left(\frac{p}{E}\right)(n-1)+1}{n}<\theta\left(\frac{p}{E}\right) .
$$

It follows that the Bertrand price $p^{B}$ is decreasing in the number of firms, and that $p^{B}>p^{e}$. The endogenous entry equilibrium must satisfy (6); we thus obtain:

$$
\frac{p^{B}-c}{p^{B}}=\frac{1+\left[\theta\left(p^{B} / E\right)-1\right] F / E L}{\theta\left(\frac{p^{B}}{E}\right)}, \quad n^{B}=\frac{E L-F}{F \theta\left(\frac{p^{B}}{E}\right)}+1 .
$$

This implies that $n^{B}>n^{e}$ and thus that excess entry is more likely in Bertrand than in monopolistic competition, a sufficient condition being (globally) $\eta^{\prime} \geqslant 0$, which includes the CES case. Notice that a competitive effect of $L$ is restored in Bertrand competition: larger markets attract more firms, which in turn strengthens competition and reduces prices. Also note that the Bertrand equilibrium converges to the monopolistic competition one for $L / F \rightarrow \infty$.

It is finally useful to notice that our Bertrand games belong to the class of aggregative games with endogenous entry analyzed in Etro (2008). Within this class of

\footnotetext{
${ }^{14}$ Cournot competition can be analysed by using the inverse demand systems derived above. Notice that in our examples this leads to "aggregative games" with multiple aggregators: see Acemoglu and Jensen (2011).
} 
games, the strategies of market leaders have no impact on the free-entry equilibrium value of $\mu$ (they only change the equilibrium number of followers). An implication of this "neutrality" result is that any firm with a first mover advantage takes $\mu$ as given and behaves according to the pricing rule (5). Therefore, these leaders choose $p^{e}$, which is lower than the price of the followers, $p^{B}$, thereby lowering the number of entrants compared to the Bertrand equilibrium case. In the case of CES preferences the welfare gains from such a lower price are exactly compensated by the losses due to the reduction in the number of varieties (Etro, 2008). When this is not the case, aggressive firms can make consumers either better off or worse off. ${ }^{15}$

\section{Conclusions}

We have studied monopolistic competition under indirect additivity, a dual assumption with respect to D-S standard setting which also encompasses a number of new analitycally tractable cases. The main properties of our market equilibrium are in contrast with those received by the literature. They coincide with those emerging under CES preferences for the impact of market size, but are entirely novel for the impact of changes in income.

Extending the present analysis to study international trade and macroeconomic fluctuations are the next natural steps. Preliminary investigations show that, in our setting, heterogenous marginal costs (à la Melitz, 2003) would create a distribution of mark up across firms wich naturally depend on the firms' size through the (passthrough) effect of a changing demand elasticity. Trade among countries of different income level should also affect the mark up distribution, possibly producing "dump-

\footnotetext{
${ }^{15}$ In particular, it can be can shown that (globally) $\eta^{\prime}>(<) 0$ implies that any price of the leaders smaller than $p^{B}$ do increase (decrease) consumer welfare.
} 
ing" (or "anti-dumping") effects. The investigation of these cases is left as a matter of future work.

\section{References}

Acemoglu, Daron and Martin Kaae Jensen, 2011, Aggregate Comparative Statics, mimeo, M.I.T

Bertoletti, Paolo and Paolo Epifani, 2012, Monopolistic Competition: CES Redux?, mimeo, University of Pavia

Bilbiie, Florin, Fabio Ghironi and Marc Melitz, 2012, Endogenous Entry, Product Variety, and Business Cycles, Journal of Political Economy, 120, 2, 304-45

Blackorby, Charles and Robert R. Russell, 1989, Will the Real Elasticicity of Substitution Plase Stand Up? (A Comparison of the Allen/Uzawa and Morishima Elasticities), The American Economic Review, 79, 882-8

Blackorby, Charles, Daniel Primont and Robert R. Russell, 1978, Duality, separability, and functional structure: theory and economic applications, North Holland

Chamberlin, Edward H., 1933, The Theory of Monopolistic Competition: A Reorientation of the Theory of Value, Harvard University Press

Dixit, Avinash and Joseph Stiglitz, 1977, Monopolistic Competition and Optimum Product Diversity, The American Economic Review, 67, 297-308

Etro, Federico, 2008, Stackelberg Competition with Endogenous Entry, The Economic Journal, 118, 1670-97

Etro, Federico and Andrea Colciago, 2010, Endogenous Market Structures and the Business Cycle, The Economic Journal, 120, 1201-34

Hicks, John, 1969, Direct and Indirect Additivity, Econometrica, 37, 2, 1969, pp. $353-4$ 
Krugman, Paul, 1979, Increasing Returns, Monopolistic Competition and International Trade, Journal of International Economics 9, pp. 469-479

Krugman, Paul, 1980, Scale Economies, Product Differentiation, and the Pattern of Trade, The American Economic Review, 70, 950-9

Melitz, Marc, 2003, The Impact of Trade on Intra-Industry Reallocations and Aggregate Industry Productivity, Econometrica, 71, 6, 1695-725

Samuelson, Paul, 1969, Corrected Formulation of Direct and Indirect Additivity, Econometrica, 37, 2, pp. 355-59

Zhelobodko, Evgeny, Sergey Kokovin, Mathieu Parenti and Jacques-François Thisse, 2012, Monopolistic Competition in General Equilibrium: Beyond the CES, Econometrica, 80, 6, 2765-84

\section{Appendix: Interpreting $\theta$ as elasticity of substitution}

In this Appendix we show that the demand elasticity $\theta$ can be interpreted as the elasticity of substitution between varieties $i$ and $j, \sigma_{i j}$, when their prices are equal (a result dual to the one which arises in the primal case: see Bertoletti and Epifani, 2012). The elasticity of substitution (see e.g. Blackorby and Russell, 1989) is given by:

$$
\sigma_{i j}=\widetilde{\varepsilon}_{j i}-\widetilde{\varepsilon}_{i i}
$$

where $\widetilde{\varepsilon}_{j i}$ is the cross elasticity of the compensated (Hicksian) demand of good $j$ with respect to the price of good $i$. The relevant compensated demands can be easily computed by using the Slutsky equation, for which: ${ }^{16}$

\footnotetext{
${ }^{16}$ In this Appendix it proves convenient to define the Marshallian demand elasticity as $\varepsilon_{i i}=$ $\partial \ln x_{i} / \partial \ln p_{i}$, rather than $-\partial \ln x_{i} / \partial \ln p_{i}$, as in the main text.
} 


$$
\widetilde{\varepsilon}_{j i}=\varepsilon_{j i}+\varepsilon_{j E} w_{i}
$$

where $\varepsilon_{j i}=\partial \ln x_{j} / \partial \ln p_{i}$ is the cross elasticity of the Marshallian demand, $\varepsilon_{j E}=$ $\partial \ln x_{j} / \partial \ln E$ is the income elasticity of good $j$ and $w_{j}=\left(p_{j} x_{j}\right) / E$ is its expenditure share. Since for (2) we can obtain:

$$
\varepsilon_{j i}=-\frac{\partial \mu}{\partial p_{i}} \frac{p_{i}}{\mu}, \quad \varepsilon_{i i}=-\theta\left(\frac{p_{i}}{E}\right)-\frac{\partial \mu}{\partial p_{i}} \frac{p_{i}}{\mu}, \quad \varepsilon_{i E}=\theta\left(\frac{p_{i}}{E}\right)-\frac{\partial \mu}{\partial E} \frac{E}{\mu},
$$

it follows that:

$$
\sigma_{i j}=\theta\left(\frac{p_{i}}{E}\right)+\left(\theta\left(\frac{p_{j}}{E}\right)-\theta\left(\frac{p_{i}}{E}\right)\right) w_{i}
$$

According to $(16), \theta(p / E)$ is equal to the elasticity of substitution between goods $i$ and $j$ if $p_{i}=p_{j}=p$ (so that $x_{i}=x_{j}$ ). Notice that in the case of a fully symmetric consumption (i.e., if $\left.p_{i}=p, i=1, \ldots, n\right) \frac{\partial \mu}{\partial p_{i}} \frac{p_{i}}{\mu}=(1-\theta(p / E)) / n, \frac{\partial \mu}{\partial E} \frac{E}{\mu}=(\theta(p / E)-$ 1) $/ n$ and

$$
\varepsilon_{j i}=\frac{\theta(p / E)-1}{n}, \quad \varepsilon_{i i}=-\frac{(n-1) \theta(p / E)}{n}-\frac{1}{n}, \quad \varepsilon_{i E}=\frac{(n-1) \theta(p / E)}{n}+\frac{1}{n} .
$$

The latter expressions are an example of the general (i.e., which holds with symmetric preferences even without additivity) relationship between the elasticity of Marshallian demand, the elasticity of substitution and the number of varieties in the case of symmetric consumption. 